\title{
Influence of maxillary incisor edge asymmetries on the perception of smile esthetics among orthodontists and laypersons
}

\author{
Andre Wilson Machado, ${ }^{\mathrm{a}}$ Won Moon, ${ }^{\mathrm{b}}$ and Luiz Gonzaga Gandini, $\mathrm{Jr}^{\mathrm{c}}$ \\ Salvador, Bahia, and Araraquara, São Paulo, Brazil, Los Angeles, Calif, Dallas, Tex, and St Louis, Mo
}

Introduction: Our objective was to determine the perception of smile esthetics among orthodontists and laypeople with respect to asymmetries on the maxillary incisor edges in a frontal smile analysis. Methods: Two frontal close-up smile photos of 2 women, 1 white and 1 Afro-Brazilian, were selected for this study. Both smiles displayed healthy maxillary anterior dentitions. The images were digitally altered to create tooth wear on the maxillary left central and lateral incisors in $0.5-\mathrm{mm}$ increments. The final images were randomly assembled into a photo album that was given to 120 judges, 60 orthodontists and 60 laypersons. Each rater was asked to evaluate the attractiveness of the images with visual analog scales. The data collected were statistically analyzed with 1-way analysis of variance with the Tukey post-hoc test and the unpaired Student $t$ test. Results: The most attractive smiles in both types of smiles were those without asymmetries and the 0.5$\mathrm{mm}$ wear in the lateral incisor. In general, tooth wear was considered unattractive by both groups of raters following a pattern: the more tooth wear, the more unattractive the smile; tooth wear in the central incisor was considered more unattractive than in the lateral incisor. For both group of raters, $0.5 \mathrm{~mm}$ of wear in the central incisor was considered unattractive, whereas the thresholds for lateral incisor discrepancies were 0.5 $\mathrm{mm}$ for orthodontists and $1.0 \mathrm{~mm}$ for laypersons. Conclusions: The result of this study corroborates the clinical assumption that symmetry between the maxillary central incisors is a paramount goal for esthetic treatments. (Am J Orthod Dentofacial Orthop 2013;143:658-64)

T: obtain optimal esthetic results, it is of paramount importance for clinicians to follow esthetic guidelines. For many years, these parameters were based only on authors' opinions rather than on evidence-based literature. ${ }^{1-6}$ These guidelines could be biased, since the concept of beauty has great subjectivity and is strongly influenced by the opinions of others. ${ }^{7-9}$ The literature suggests that orthodontists and laypeople have different perceptions of smile

\footnotetext{
${ }^{a}$ Associate professor, Section of Orthodontics, Dental School, Federal University of Bahia, Salvador, Bahia, Brazil.

${ }^{\mathrm{b}}$ Program director, Section of Orthodontics, School of Dentistry, University of California at Los Angeles, Calif.

'Professor, School of Dentistry, Paulista State University, Araraquara, São Paulo, Brazil; adjunct clinical professor, Baylor College of Dentistry, Dallas, Tex, and Saint Louis University, St Louis, Mo.

The authors report no commercial, proprietary, or financial interest in the products or companies described in this article.

Funding from CAPES/Brazil Bex: 4832/10-8.

Reprint requests to: Andre Wilson Machado, Av. Araújo Pinho, 62, 7 Andar, Canela, Salvador/BA - Brazil, CEP. 40.110-150; e-mail, awmachado@gmail. com.

Submitted, November, 2012; revised and accepted, January, 2013. 0889-5406/ $\$ 36.00$

Copyright (C) 2013 by the American Association of Orthodontists.

http://dx.doi.org/10.1016/j.ajodo.2013.02.013
}

esthetics when evaluating a variety of orofacial characteristics, and that orthodontists are more sensitive in detecting deviations from ideal than the general public. ${ }^{10-13}$

To provide more objective guidelines regarding the perception of smile esthetics, numerous studies were performed by using digital-image manipulations. ${ }^{10-19}$ Thereby, some smile characteristics were better elucidated: smile arc, ${ }^{10,14-16}$ amount of gingival display, ${ }^{11,12,14,17}$ type of buccal corridors, ${ }^{10,15,16}$ presence of dental and gingival asymmetries, ${ }^{11-14}$ presence of a midline diastema, ${ }^{12,16,18}$ influence of midline and long axis deviations, ${ }^{11,13,14}$ and importance of maxillary incisor sizes and proportions. ${ }^{15,18,19}$

According to the literature, an esthetic treatment plan must begin at the maxillary central incisor area ${ }^{1,3,6}$; thus, dental or gingival asymmetries must be carefully analyzed. ${ }^{11-14}$ The impact of gingival asymmetries on the perception of smile esthetics is well documented in the literature. When there is a gingival margin discrepancy between the central and lateral incisors, neither laypersons nor dental professionals considered a 2-mm discrepancy unesthetic. ${ }^{12,14}$ However, when 

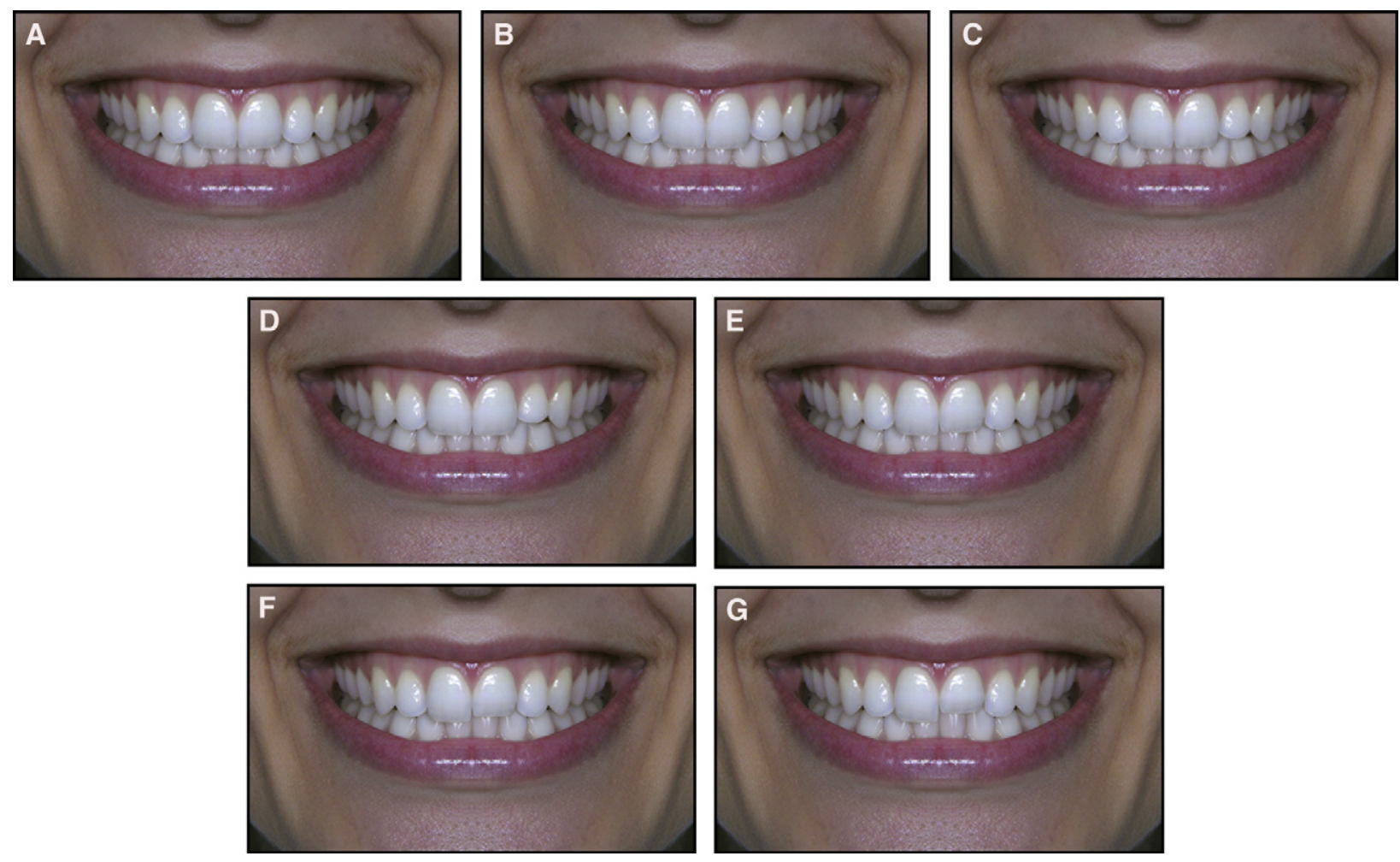

Fig 1. Tooth wear in a white woman in 0.5-mm increments: A, control; $\mathbf{B}, 0.5 \mathrm{~mm}$, lateral incisor; C, 1.0 $\mathrm{mm}$, lateral incisor; $\mathbf{D}, 1.5 \mathrm{~mm}$, lateral incisor; $\mathbf{E}, 0.5 \mathrm{~mm}$, central incisor; $\mathbf{F}, 1.0 \mathrm{~mm}$, central incisor; $\mathbf{G}$, $1.5 \mathrm{~mm}$, central incisor.

the gingival margin discrepancy was between the central incisors, slight discrepancies were considered unattractive by orthodontists and laypersons. ${ }^{12,13}$ Although this confirms that symmetry between the maxillary central incisors is an important aspect, it also highlights a question: if small gingival asymmetries are not recognized by laypeople as unattractive, is it necessary to treat?

After we analyzed this information, other questions arose. If an asymmetry is related to the maxillary incisor edges, what do laypeople and orthodontists perceive? In other words, what is the threshold for these people when evaluating uneven central and lateral incisors with tooth wear? This information is of paramount importance because it can assist the orthodontic clinician during the finishing and detailing phases. For instance, Kokich et $\mathrm{al}^{12}$ stated that if a dental asymmetry is not recognized as unesthetic, it might not be necessary to commit the patient to restorations.

Those questions were explored in this study, with the objective to determine the perception of smile esthetics among orthodontists and laypeople with respect to asymmetries on the maxillary incisor edges in a frontal smile analysis. The null hypothesis tested was that these asymmetries would be equally rated as attractive by orthodontists and laypeople.

\section{MATERIAL AND METHODS}

According to a pilot study, a sample size calculation was undertaken by using software (version 5.0; BioStat, Mamirauá Institute, Tefé, Amazonas, Brazil). On the basis of a significance level of alpha 0.01 and the effect size estimated at 0.95 , the sample size was calculated to achieve $80 \%$ power. This calculation showed that 58 subjects in each group were necessary.

Two standardized frontal photos of pleasant smiles of a white woman and an Afro-Brazilian woman were selected for this study. Both women had unworn, unrestored, and healthy maxillary incisors and had not had orthodontic treatment. These smiles were considered highly attractive and followed some principles of an ideal smile described in the literature: adequate width-to-length proportion of the esthetic zone, convex smile arc, gingival display less than $1.0 \mathrm{~mm}$, gingival line of the central incisor matching the canine and the lateral incisor slightly below, and progressive increases in the depth of tooth embrasures from the central incisor to the canine. ${ }^{1-6}$ 

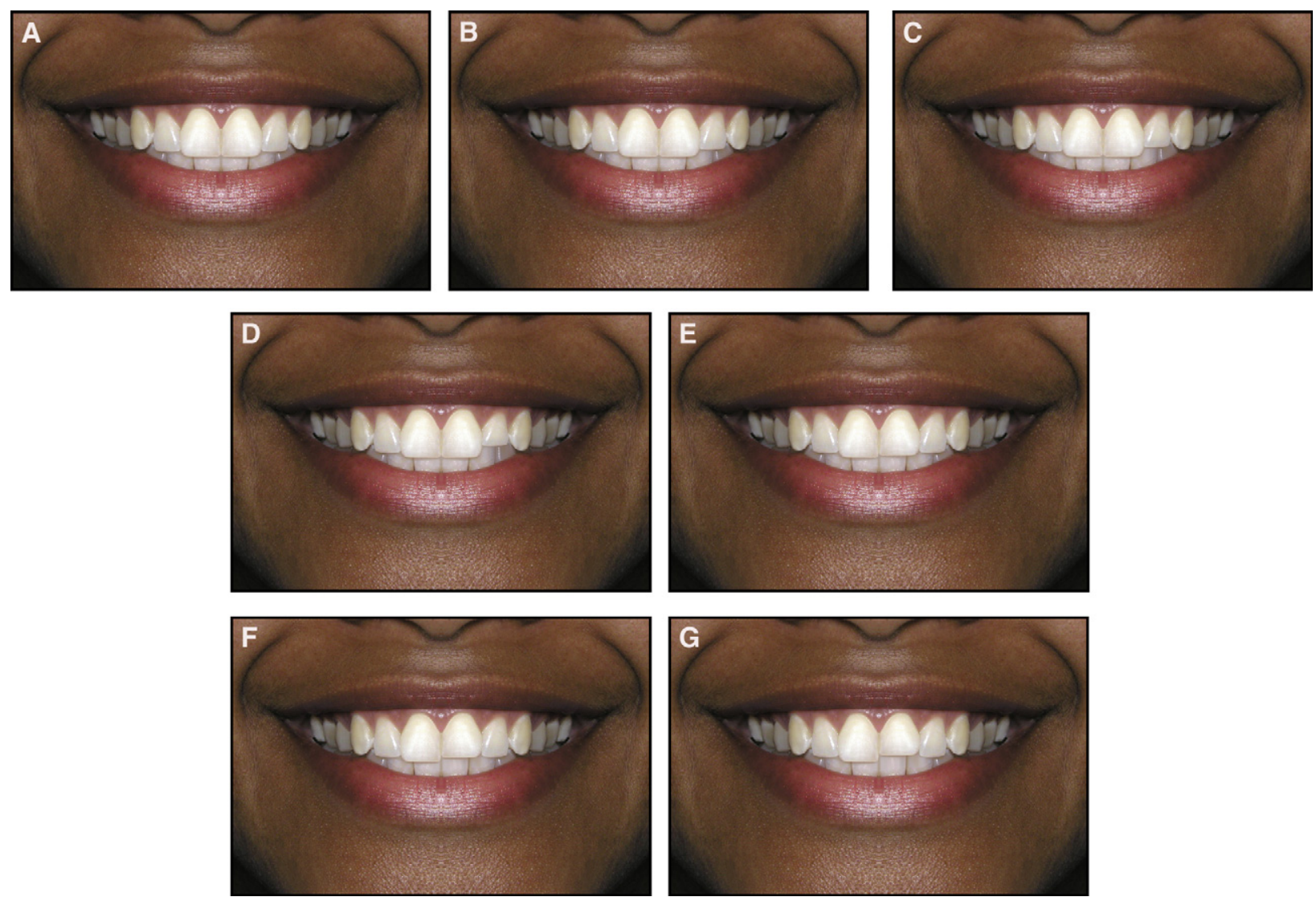

Fig 2. Tooth wear in an Afro-Brazilian woman in 0.5- $\mathrm{mm}$ increments: $\mathbf{A}$, control; $\mathbf{B}, 0.5 \mathrm{~mm}$, lateral incisor; C, $1.0 \mathrm{~mm}$, lateral incisor; $\mathbf{D}, 1.5 \mathrm{~mm}$, lateral incisor; $\mathbf{E}, 0.5 \mathrm{~mm}$, central incisor; $\mathbf{F}, 1.0 \mathrm{~mm}$, central incisor; $\mathbf{G}, 1.5 \mathrm{~mm}$, central incisor.

The selected images were digitally altered by using Adobe Photoshop (CS3; Adobe Systems, San Jose, Calif). The photos were manipulated to produce symmetrical images and were then retouched to adjust color, brightness, and contrast, as well as to remove any discolorations on the lips and skin. Each image was then condensed to achieve an image with measurements identical to those on the actual patient. Thus, each millimeter measured on the digital and printed image was equivalent to each millimeter measured clinically on the patient, with the maxillary central incisor as the reference. Furthermore, after recommendations from previous studies, much of the nose and chin was removed to reduce the number of variables in the images. ${ }^{11-19}$

Each new image was altered in 0.5- $\mathrm{mm}$ increments on the incisor edges of the maxillary left central and lateral incisors. In all images, the gingival margins, the papillary heights, and the right sides of the image were not altered. For both photos, 7 new images were created (Figs 1 and 2).
The final images were digital files with a resolution of $300 \mathrm{dpi}$. They were professionally printed with specialized digital equipment (Minilab Digital Frontier 570; Fuji Film, Manaus, Amazonas, Brazil) on standard A4 size format $(29.7 \times 42 \mathrm{~cm})$ Kodak Edge Generations paper (Kodak do Brasil, Manaus, Amazonas, Brazil). Then a photo album was assembled containing all images from each group in random order.

The album was given to 120 judges, 60 orthodontists ( 37 men, 23 women) and 60 laypeople ( 32 men, 28 women) with a college education but no dental background. Each rater was given brief information about the study and asked to evaluate the attractiveness of the images. Along with the album, each judge received a form with 100-mm visual analog scales printed for each image, as in previous studies. ${ }^{10,13-17}$ The scale ranged from "very unattractive" on the far left to "very attractive" at the far right. A line was also printed at the midpoint of each scale to provide a reference line for an average level of attractiveness. All judges marked a point along the scale according 
Table I. Orthodontists' and laypersons' perceptions of smiles of a white woman

White woman

\begin{tabular}{|c|c|c|c|c|c|c|c|}
\hline \multirow[b]{2}{*}{ Altered asymmetry } & \multicolumn{3}{|c|}{ Orthodontists } & \multicolumn{3}{|c|}{ Laypersons } & \multirow[b]{2}{*}{ Difference } \\
\hline & Mean & $S D$ & Results* & Mean & $S D$ & Results* & \\
\hline No asymmetry & 86.00 & 9.99 & A & 87.26 & 9.71 & A & \\
\hline $0.5 \mathrm{~mm}$, lateral incisor & 78.20 & 8.45 & A & 87.15 & 8.31 & A & $\dagger$ \\
\hline $1.0 \mathrm{~mm}$, lateral incisor & 51.64 & 16.2 & B & 80.43 & 13.89 & $A, B$ & $\dagger$ \\
\hline $1.5 \mathrm{~mm}$, lateral incisor & 37.04 & 16.05 & $\mathrm{C}$ & 61.91 & 10.92 & $\mathrm{C}$ & $\dagger$ \\
\hline $0.5 \mathrm{~mm}$, central incisor & 51.49 & 13.33 & B & 72.44 & 9.25 & B & † \\
\hline $1.0 \mathrm{~mm}$, central incisor & 22.35 & 10.82 & D & 29.34 & 14.29 & D & $\dagger$ \\
\hline $1.5 \mathrm{~mm}$, central incisor & 9.21 & 3.7 & E & 22.36 & 12.21 & D & $\dagger$ \\
\hline
\end{tabular}

*Smiles with the same letter did not differ from each other $(P<0.05)$; ${ }^{\dagger}$ statistical difference between the 2 group of raters $(P<0.05)$.

Table II. Orthodontists' and laypersons' perceptions of smiles of an Afro-Brazilian woman

Afro-Brazilian woman

\begin{tabular}{|c|c|c|c|c|c|c|c|}
\hline \multirow[b]{2}{*}{ Altered asymmetry } & \multicolumn{3}{|c|}{ Orthodontists } & \multicolumn{3}{|c|}{ Laypersons } & \multirow[b]{2}{*}{ Difference } \\
\hline & Mean & $S D$ & Results* & Mean & $S D$ & Results* & \\
\hline No asymmetry & 83.41 & 11.20 & A & 87.61 & 8.74 & A & \\
\hline $0.5 \mathrm{~mm}$, lateral incisor & 80.40 & 9.92 & A & 86.16 & 7.26 & A & $\dagger$ \\
\hline $1.0 \mathrm{~mm}$, lateral incisor & 55.72 & 10.64 & B & 80.32 & 8.79 & A, B & $\dagger$ \\
\hline $1.5 \mathrm{~mm}$, lateral incisor & 30.11 & 13.70 & C & 50.38 & 12.61 & C & $\dagger$ \\
\hline $0.5 \mathrm{~mm}$, central incisor & 57.66 & 16.17 & B & 72.52 & 11.35 & B & $\dagger$ \\
\hline $1.0 \mathrm{~mm}$, central incisor & 24.04 & 14.45 & C, D & 29.18 & 14.32 & D & \\
\hline $1.5 \mathrm{~mm}$, central incisor & 16.05 & 12.89 & $\mathrm{D}$ & 23.98 & 15.00 & D & $\dagger$ \\
\hline
\end{tabular}

*Smiles with the same letter did not differ from each other $(P<0.05)$; ${ }^{\dagger}$ statistical difference between the 2 group of raters $(P<0.05)$.

to their perceptions of smile esthetics. The scores were then measured in millimeters by the first author with an electronic digital caliper (Starrett, Suzhou, China).

To assess the reliability of the method, 6 raters from each group were randomly selected. They were asked to evaluate 1 page of the album on which there were 2 identical images. Correlation coefficients were used to compare the scores for those images to determine intrarater agreement. High levels of reliability were found, since all coefficients were greater than or equal to 0.76 for both groups of raters.

\section{Statistical analysis}

The data were statistically analyzed with SPSS software (version 16.0; SPSS, Chicago, 111). Descriptive statistics were reported as means and standard deviations. Differences in the mean esthetic scores in the levels of asymmetries were analyzed by using 1-way analysis of variance with the Tukey post-hoc test. To compare the distributions of the mean scores between orthodontists and laypersons, the unpaired Student $t$ test was used. The level of signiflcance was established at 5\%.

\section{RESULTS}

From the orthodontists' standpoint, the most attractive smiles among the images of the white woman were the symmetrical smile (mean, 86.0) and the 0.5- $\mathrm{mm}$ asymmetry in the lateral incisor (mean, 78.20); the least attractive was the $1.5-\mathrm{mm}$ wear at the central incisor (mean, 9.27). From the laypersons' opinions, the most attractive smiles were the symmetrical smile (mean, 87.26), the 0.5-mm wear on the lateral incisor (mean, 87.15), and the 1.0-mm wear on the lateral incisor (mean, 80.43); the least attractive smiles were those with $1.0 \mathrm{~mm}$ and $1.5 \mathrm{~mm}$ of wear on the central incisors (means, 29.34 and 22.36, respectively) (Table 1).

Similar results were found for the Afro-Brazilian woman's smile. For the orthodontists, the most attractive smiles were the symmetrical smile (mean, 83.41) and the 0.5-mm wear on the lateral incisor (mean, 80.40), and the least attractive were those with $1.0 \mathrm{~mm}$ and $1.5 \mathrm{~mm}$ of wear on the central incisor (mean, 24.04 and 16.05, respectively). For the laypersons, the most attractive smiles were the symmetrical (mean, 87.61), the 0.5-mm wear on the lateral incisor (mean, 86.16), and the 1.0-mm wear on the lateral 
incisor (mean, 80.32); the least attractive were the smiles with wear of $1.0 \mathrm{~mm}$ and $1.5 \mathrm{~mm}$ on the central incisor (means, 29.18 and 23.98, respectively) (Table 11).

When comparing the perceptions of the orthodontists and laypersons, they showed statistical differences in most situations, with the latter group giving higher scores $(P<0.05)$. In general, both groups had similar ratings for the most attractive smile, which was the symmetrical one (Tables 1 and 11$)$.

\section{DISCUSSION}

Dental and gingival asymmetries in the esthetic zone are common problems in adult patients. These situations are mainly caused by tooth wear or abrasion of the incisors, causing unequal crown lengths. Because an active incisor eruption can be followed by uneven tooth wear, gingival asymmetry might also occur. According to the literature, the treatment of dental asymmetries is a simple procedure; depending on the location and severity of the problem, it can be accomplished by enamel reshaping, composite restorations, or porcelain veneers. ${ }^{6,20,21}$ If, after restoring the tooth anatomy, a gingival asymmetry is still present, it can be corrected by periodontal surgery or orthodontic intrusion or extrusion complemented by composite restorations or tooth enamel reshaping. ${ }^{22,23}$

Although those treatment strategies are well documented in the literature, from an esthetic standpoint, an intriguing question can be asked: is it necessary to correct? In other words, if laypeople cannot recognize a dental or gingival asymmetry as unattractive, why should dental specialists need to treat it? As discussed before, slight gingival margin discrepancies in the maxillary anterior dentition cannot be recognized as unesthetic by laypersons or dental professionals. ${ }^{12-14}$ Those results suggest that the treatment of slight gingival discrepancies might reflect an exaggerated concern by dental specialists rather than an esthetic need. ${ }^{13}$ It can also be stated that the threshold depends not only on the group of raters, but also on the location of the asymmetry. Gingival discrepancies farther from the midline are more tolerated than those closer to the midline.

The methodology used by those authors to assess the influence of crown-length discrepancies in the esthetic perception of smiles modified the position of the gingival margins. ${ }^{11-14}$ In this way, the main variable studied was the impact of gingival asymmetries in the perception of smile esthetics. On the other hand, in our study, the variable studied was not the influence of gingival asymmetries but the impact of incisal discrepancies on the perception of smile esthetics.

The results of our study showed that even a slight incisal discrepancy of $0.5 \mathrm{~mm}$ between the maxillary central incisors was rated as unattractive by laypeople and orthodontists. This finding supports the clinical assumption that symmetry between the maxillary central incisors is of paramount importance and also that these teeth are the key to evaluating smile esthetics. ${ }^{1,3,6}$ It is also important to consider that laypeople were sensitive in detecting dental asymmetries in our study compared with gingival asymmetries in previously published studies. ${ }^{1-14}$ Based on those studies, from the laypeople's perceptions, correction of a gingival asymmetry of 1.0 to $1.5 \mathrm{~mm}$ between the maxillary central incisors might not be necessary, but a $0.5-\mathrm{mm}$ incisal edge discrepancy might be. Therefore, the clinician should refer orthodontic patients for restoration of uneven central incisors not only to reestablish anterior guidance and prevent active incisor eruption, but also to optimize smile esthetics.

Although the thresholds for orthodontists and laypeople were similar, their behaviors for the discrepancies on the lateral incisor were statistically different. The thresholds were $0.5 \mathrm{~mm}$ for orthodontists and $1.0 \mathrm{~mm}$ for laypeople. Following the same idea addressed before, correction of a 1.0-mm incisal discrepancy between the maxillary lateral incisors might reflect an excessive concern by dental specialists rather than an esthetic need.

Pinho et $\mathrm{al}^{13}$ found that asymmetrical wear of $2.0 \mathrm{~mm}$ on a canine cusp had no impact on the perception of smile esthetics. In this way, it can be hypothesized that incisal asymmetries on the maxillary canines are more tolerated by orthodontists and laypeople, followed by the maxillary lateral and central incisors. This finding corroborates a clinical assumption that the closer to the midline, the greater the need of symmetry, and the farther from the midline, more gentle asymmetries are acceptable. $^{6}$

Therefore, the clinician should first locate and measure the amount of tooth wear, and then decide whether a restoration is necessary. Kokich et al ${ }^{12}$ noted that in some situations if slight deviations are not recognized as unattractive, why refer the patient for cosmetic restorations that would eventually need to be replaced? In addition, studies have shown that a composite restoration accumulates more biofilm than natural enamel and also is more prone to discolorations with time. ${ }^{24,25}$

With this in mind, in a clinical situation of a crown length discrepancy, the clinician should give more priority to correcting the incisal discrepancy than the gingival asymmetry. Based on our data and previous studies, orthodontists and laypeople are more tolerant of gingival asymmetries than incisal-edge discrepancies. ${ }^{11-14}$ Thus, if a gingival asymmetry is within the patient's threshold, it makes more sense to 
match the incisal edges by simple procedures such as enamel reshaping and restorations than to commit the patient for periodontal surgery or even a multidisciplinary approach involving orthodontics and periodontics.

We surveyed orthodontists and laypeople. The first group was selected because previous studies showed that they are the most sensitive in detecting deviations from ideal. ${ }^{10-13,15,17}$ The latter group was chosen because they are the primary consumers of dental services, as opposed to practitioners, who are the providers of care. ${ }^{14}$ Following the tendency, these groups of raters had different perceptions. In most situations, orthodontists were more critical in their evaluations. However, for the symmetrical smiles, both groups displayed no statistical difference. It can be hypothesized that an ideal smile arrangement can easily be recognized as attractive by any group of raters. In contrast, when small deviations occur, they start to show differences in their judgments.

In previous studies, the smiles used for evaluation were from white female patients. ${ }^{11-15,19}$ For this reason, we also included a smile from an AfroBrazilian woman. The main reason for evaluating this smile was to survey possible differences in the influence of asymmetries in the esthetic perceptions. The results of our study suggest that the behaviors of orthodontists and laypeople were similar when the smiles of the white and Afro-Brazilian women were compared. Although the objective was not to compare those types of smiles, it can be stated that the impact of dental asymmetries in both situations was similar.

Since we used computer-manipulated images from 2 patients and the opinions of specific groups, the results should be carefully analyzed. As stated by Kokich et al, ${ }^{12}$ since the results and conclusions are based on averages, it is difficult to customize this information to a patient because of the subjectivity of evaluations of smile esthetics. Therefore, we support their suggestion to discuss the results of this study with patients who have a dental asymmetry and then decide whether to treat it or leave it.

\section{CONCLUSIONS}

The outcomes of this study demonstrate the following.

1. The most attractive smiles in both types of images (white and Afro-Brazilian women) were those without asymmetries or with a $0.5-\mathrm{mm}$ asymmetry on the lateral incisor.

2. Tooth wear was considered unattractive following a pattern: the greater the tooth wear, the more unattractive the smile; tooth wear on the central incisor was considered more unattractive than tooth wear on the lateral incisor.

3. For both groups of raters, $0.5 \mathrm{~mm}$ of wear on the central incisor was considered unattractive, whereas the thresholds for lateral incisor discrepancies were $0.5 \mathrm{~mm}$ for orthodontists and $1.0 \mathrm{~mm}$ for laypersons.

\section{REFERENCES}

1. Lombardi RE. The principles of visual perception and their clinical application to denture esthetics. J Prosthet Dent 1973;29:358-82.

2. Tjan AH, Miller GD, The JG. Some esthetic factors in a smile. J Prosthet Dent 1984;51:24-8.

3. Rufenacht CR. Fundamentals of esthetics. Chicago: Quintessence; 1990.

4. Peck S, Peck L. Selected aspects of the art and science of facial esthetics. Semin Orthod 1995;1:105-26.

5. Garber DA, Salama MA. The aesthetic smile: diagnosis and treatment. Periodontol 2000 1996;11:18-28.

6. Chiche G, Pinault A. Esthetics of anterior fixed prosthodontics. Chicago: Quintessence; 1994.

7. Van der Geld P, Oosterveld P, Heck GV, Kuijpers-Jagtman AM. Smile attractiveness: self-perception and influence on personality. Angle Orthod 2007;77:759-75.

8. Mcleod C, Fields HW, Hechter F, Wiltshire W, Rody W, Christensen J. Esthetics and smile characteristics evaluated by laypersons: a comparison of Canadian and US data. Angle Orthod 2011;81:198-205.

9. Owens EG, Goodacre CJ, Loh PL, Hanke G, Okamura M, Jo KH, et al. A multicenter interracial study of facial appearance. Part 2: a comparison of intraoral parameters. Int J Prosthodont 2002; 15:283-8.

10. Parekh SM, Fields HW, Beck M, Rosenstiel S. Attractiveness of variations in the smile arc and buccal corridor space as judged by orthodontists and laymen. Angle Orthod 2006;76:557-63.

11. Kokich VO, Kiyak HA, Shapiro PA. Comparing the perception of dentists and lay people to altered dental esthetics. J Esthet Dent 1999;116:311-24.

12. Kokich VO, Kokich VG, Kiyak HA. Perceptions of dental professionals and laypersons to altered dental esthetics: asymmetric and symmetric situations. Am J Orthod Dentofacial Orthop 2006;130:141-51.

13. Pinho S, Ciriaco C, Faber J, Lanza MA. Impact of dental asymmetries on the perception of smile esthetics. Am J Orthod Dentofacial Orthop 2007;132:748-53.

14. Ker AJ, Chan R, Fields HW, Beck M, Rosenstiel S. Esthetics and smile characteristics from the layperson's perspective: a computer-based survey study. J Am Dent Assoc 2008;139:1318-27.

15. Krishnan V, Daniel ST, Lazar D, Asok A. Characterization of posed smile by using visual analog scale, smile arc, buccal corridor measures, and modified smile index. Am J Orthod Dentofacial Orthop 2008;133:515-23.

16. Rodrigues CDP, Magnani R, Machado MS, Oliveira OB. The perception of smile attractiveness. Angle Orthod 2009;79:634-9.

17. Suzuki L, Machado AW, Bittencourt MAV. An evaluation of the influence of gingival display level in the smile esthetics. Dent Press J Orthod 2011;165:37-9.

18. Rosenstiel SF, Rashid RG. Public preferences for anterior tooth variations: a web-based study. J Esthet Restor Dent 2002;142:97-106. 
19. Cooper GE, Tredwin CJ, Cooper NT, Petrie A, Gill DS. The influence of maxillary central incisor height-to-width ratio on perceived smile aesthetics. Br Dent J 2012;212:589-99.

20. Sarver DM. Principles of cosmetic dentistry in orthodontics: part 1. Shape and proportionality of anterior teeth. Am J Orthod Dentofacial Orthop 2004;126:749-53.

21. Brehm TW. Improving esthetics by recontouring natural teeth. J Prosthet Dent 1971;26:497-500.

22. Kokich VG. Anterior dental esthetics: an orthodontic perspective 1. Crown length. J Esthet Dent 1993;5:19-23.
23. Reddy MS. Achieving gingival esthetics. J Am Dent Assoc 2003; 134:295-304

24. Montanaro L, Campoccia D, Rizzi S, Donati ME, Breschi L, Prati C, et al. Evaluation of bacterial adhesion of Streptococcus mutans on dental restorative materials. Biomaterials 2004;25: 4457-63.

25. Lima FG, Romano AR, Correa MB, Demarco FF. Influence of microleakage, surface roughness and biofilm control on secondary caries formation around composite resin restorations: an in situ evaluation. J Appl Oral Sci 2009;17:61-5. 